\title{
Sacrificio cortoplacista adaptativo 2opt (SCA_2opt): Una heuristica inspirada en el pensamiento sistémico
}

\author{
Jorge Pérez Rave ${ }^{\mathrm{a} *}$, Patricia Jaramillo Álvarez ${ }^{\mathrm{b}}$ \\ a*jiprave@gmail.com, UdeA, Colômbia \\ bypjarami@unal.edu.co, UNAL, Colômbia
}

\begin{abstract}
Resumen
Se detalla el origen de dos noveles heurísticas para el TSP simétrico, inspiradas en el pensamiento sistémico: Sacrificio Cortoplacista Adaptativo 2-opt (SCA_2opt) y SCA_2_opt_r. Estas surgen del análisis sistémico de la regla de decisión Vecino más cercano, identificándosele el arquetipo "Soluciones contraproducentes". El SCA se basa en que el agente viajero renuncie en un momento dado a una ciudad inmediatamente cercana, y se traslade hacia la segunda ciudad más cercana disponible. A partir de ello, se continúa con la regla del vecino más cercano. Cada que se realiza el SCA (búsqueda global) se efectúa una búsqueda local 2_opt. Considerando el binomio eficacia y eficiencia, las dos heurísticas se muestran prometedoras en comparación multicriterio contra 19 metaheurísticas. Se evidencia que el pensamiento sistémico es un campo de inspiración viable para el desarrollo de métodos de optimización combinatoria; se plasman preguntas emergentes para desarrollos futuros, que permitan continuar integrando elementos de la optimización clásica con el pensamiento sistémico; áreas tradicionalmente vistas como antagónicas, pero cuyo diálogo se muestra favorable en este artículo.
\end{abstract}

Palabras clave

Sacrificio cortoplacista adaptativo. TSP. Metaheurísticas. Análisis multicriterio. Pensamiento sistémico.

\section{Introducción}

La optimización combinatoria es una rama de la programación matemática que reúne una serie de problemas en los que las variables toman valores discretos, los cuales se combinan para encontrar soluciones óptimas en un conjunto finito de soluciones (MORALES et al., 2011). En este campo de la investigación de operaciones existen problemas conocidos como NP-hard, en los cuales no es viable, desde el punto de vista práctico, explorar todas las posibles combinaciones para encontrar la solución óptima, ya que su identificación podría consumir generaciones humanas respecto al tiempo computacional. De ahí la necesidad de emplear estrategias heurísticas que no garantizan la localización de la solución óptima, pero sí, con viabilidad práctica, una posible cercanía a ella.

A través de la historia, los avances en la optimización combinatoria han sido motivados, en su mayoría, por la búsqueda de soluciones heurísticas a un problema de prueba tipo NP-hard, conocido como el "Problema del Vendedor Viajero" o TSP (Traveling Salesman Problem). Sobre ello se encuentran trabajos iniciales como Dantzig, Fulkerson y Jhonson, (1954) y Flood (1956), y otros más recientes, entre ellos: Nguyen et al. (2007) y Whitley, Hains y Howe (2010).

El TSP representa un agente para el que se desea conocer ¿cómo debe elaborar su plan de viaje de modo que visite cada ciudad exactamente una vez, regrese al punto de origen y genere el menor costo posible? (FLOOD, 1956).

Los aportes noveles a la resolución de este problema se han inspirado en diversos fenómenos observables en la realidad, como por ejemplo, la evolución biológica (algoritmos genéticos: CARTER; RAGSDALE, 2006; WHITLEY; HAINS; HOWE, 2010), el comportamiento de hormigas reales (DORIG0; GAMBARDELLA, 1995, 1997) y el de abejas (KARABOGA; GORKEMLI, 2011), entre muchos otros. Mayor información sobre ello puede encontrarse en Pérez (2011a), quien concluye que, hoy día, para mover las puntas actuales de conocimiento 
hacia esferas desconocidas que trasciendan la generación de híbridos de los métodos ya existentes, es preciso introducir nuevos campos de inspiración que incorporen otras perspectivas, posibilitando la creación de noveles métodos heurísticos.

Haciendo énfasis en los posibles campos de inspiración, precisamente hay una disciplina que viene cobrando cada vez más relevancia como necesidad y tendencia en los métodos de análisis y solución de problemas sociales, políticos y empresariales (SENGE, 1998, PÉREZ, 2011b), así como en las competencias genéricas exigidas para los profesionales de ingeniería (LETELIER et al., 2005; DISTEFANO; HAARTH; IRIARTE, 2006). Se trata del pensamiento sistémico (PS), el cual busca "[...] comprender y describir las fuerzas y las interrelaciones que dan forma al comportamiento del sistema [...]" (BAHILL; GISSING, 1998), a fin de posteriormente intervenirlo con soluciones efectivas y duraderas. A pesar de las posibilidades que ofrece esta disciplina, la revisión previa sobre el TSP (PÉREZ, 2011a) evidenció ausencia del uso del PS como campo de inspiración para el desarrollo de métodos de optimización combinatoria.

Ante esa posibilidad de trabajo fututo, el presente estudio se enfocó en probar el pensamiento sistémico (PS) como un posible nuevo campo de inspiración para el desarrollo de noveles métodos de optimización combinatoria, delimitando al TSP simétrico como referente de prueba que es.

Además de la efectividad del PS para solucionar problemas complejos, la intención de explorarlo como posible campo de inspiración en la optimización combinatoria se hace más pertinente, teniendo presente que la relación entre la optimización clásica ("ésta es la solución óptima”) y el pensamiento sistémico ("depende de la perspectiva"), en casos, puede ser interpretada como antagónica, pero que en recientes trabajos se muestra prometedor el posible diálogo entre ellos, por ejemplo, en Pérez (2011b).

Bajo este contexto, el problema de investigación al que se ha delimitado este artículo, fue sistematizado mediante la siguiente pregunta: ¿Un algoritmo heurístico inspirado en el análisis sistémico del "Vecino más cercano", es capaz de arrojar soluciones efectivas (eficaces y eficientes) para instancias simétricas TSP?

Haciendo énfasis en el alcance del estudio, éste se ha delimitado a explorar el uso del pensamiento sistémico en la regla de decisión vecino más cercano (VMC), como primer objeto de análisis. Ello se debió a que el VMC es un método clásico en el TSP, usado con frecuencia para el inicio de otros algoritmos (JÜNGER; REINELT; RINALDI, 1995; LIU, 2007; GÓMEZ; LEÓN, 2011), al cual no se le ha dado una interpretación desde las leyes del pensamiento sistémico. Esto puede ser pertinente, puesto que la regla del VMC parte del sentido común, se destaca por su eficiencia computacional, a costa del deterioro en la respuesta arrojada (BENTLEY, 1992; MARTI, 2003). Solo se enfoca en visitar, cada vez, la ciudad inmediatamente cercana disponible, sin considerar las afectaciones que ello pueda generar en posteriores visitas del viajero. Para profundizar en el referencial teórico remítase a Pérez (2011a), apartado de revisión sistemática de literatura para el TSP.

En la sección 2 se presentan los materiales y métodos, la sección 3 ofrece los resultados acompañados de la discusión, la sección 4 trata las conclusiones del estudio y la sección 5 los trabajos futuros.

Tabla 1. Momentos metodológicos seguidos.

\begin{tabular}{|l|l|l|}
\hline \multicolumn{1}{|c|}{ Etapas } & \multicolumn{1}{|c|}{ Actividades } & \multicolumn{1}{c|}{ Descripción } \\
\hline \multirow{4}{*}{$\begin{array}{l}\text { La regla del VMC vista } \\
\text { desde el pensamiento } \\
\text { sistémico }\end{array}$} & $\begin{array}{l}\text { Introducción en el campo de } \\
\text { conocimiento sobre el TSP }\end{array}$ & $\begin{array}{l}\text { Estado actual del conocimiento sobre el TSP y de sus oportunidades de } \\
\text { investigación. }\end{array}$ \\
\cline { 2 - 3 } & $\begin{array}{l}\text { Revisión de arquetipos y leyes del } \\
\text { pensamiento sistémico }\end{array}$ & $\begin{array}{l}\text { Apoyados en “La quinta disciplina” y sus diferentes versiones se profundizó en el } \\
\text { conocimiento sobre el posible campo de inspiración a someter a prueba. }\end{array}$ \\
\cline { 2 - 3 } $\begin{array}{l}\text { Análisis de la heurística VMC } \\
\text { para el TSP, bajo una perspectiva } \\
\text { de sistemas. }\end{array}$ & $\begin{array}{l}\text { El comportamiento de las soluciones arrojadas por el VMC para el TSP, se analizó } \\
\text { bajo las leyes del pensamiento sistémico, buscando identificar alguna estructura } \\
\text { sistémica subyacente. }\end{array}$ \\
\hline $\begin{array}{l}\text { Diseño y desarrollo del } \\
\text { método heurístico a } \\
\text { proponer }\end{array}$ & Diseño & $\begin{array}{l}\text { Con base en la etapa anterior y siguiendo el pensamiento lógico: observar, } \\
\text { comprender, actuar, mejorar, se diseño la heurística, buscando que a través de un } \\
\text { punto de apalancamiento, pudiese generarse una alteración leve a la heurística } \\
\text { del VMC, en busca de un mejor desempeño. }\end{array}$ \\
\cline { 2 - 3 } Experimentación & Programación & $\begin{array}{l}\text { Realizado en Visual Basic bajo Macros de Excel } \\
\text { se establecieron las siguientes variables respuesta: costo medio (CM) del tour, } \\
\text { mejor costo (MC) encontrado, número de tours (NT) empleados para arrojar el } \\
\text { mejor costo, y tiempo medio (TM). En casos, esto último se reforzó con una } \\
\text { aproximación a gigaciclos de procesador (GCP), tomando el "peor caso" para la } \\
\text { heurística a desarrollar. }\end{array}$ \\
\hline
\end{tabular}




\section{Materiales y métodos}

En la Tabla 1 se resumen las etapas, las actividades y la descripción de las mismas. Todo ello representa los principales momentos metodológicos seguidos.

La comparación bajo análisis multicriterio se consideró pertinente, puesto que la literatura reporta lo vital de que un método de optimización heurística presente diversas propiedades, entre ellas, amigabilidad, sencillez, eficacia y eficiencia (HANSEN; MLADENOVIC, 2003), siendo estas dos últimas las de interés en este artículo, debido a su viabilidad de análisis objetivo.

Otro de los aspectos que llevaron a efectuar análisis multicriterio fue la posibilidad de incorporar pesos a los factores a considerar, más aún cuando en la optimización combinatoria y hasta en diversos campos empresariales, eficacia y eficiencia suelen ser objetivos contradictorios. En ese sentido, el estudio consideró cinco escenarios de importancia relativa para eficacia y eficiencia: equivalentes a 50\%; apoyados en el principio de Pareto 80\%-20\% (vital, trivial) y viceversa; $y$ casos extremistas donde el decisor centra con exageración su inclinación hacia un solo factor (100\% de importancia), dejando el otro nulo. En la Tabla 2 se detallan las metaheurísticas que sirvieron de base comparativa, las cuales fueron elegidas buscando incluir trabajos de diversas familias de algoritmos y ámbitos geográficos.

Así mismo, para las comparaciones multicriterio otro de los lineamientos fue que los trabajos elegidos arrojaran información sobre el binomio eficacia y eficiencia de los métodos que probaron o citaron.

\section{Resultados y discusión}

\subsection{La regla del vecino más cercano vista desde el pensamiento sistémico}

\subsubsection{Historia de don Pablo (Adaptado de PÉREZ, 2011a)}

Suponga que don Pablo ha programado unas vacaciones y cuenta con 337 millones de pesos para visitar 130 ciudades. Con el fin de que el dinero le alcance para su plan vacacional, don Pablo, recurriendo al sentido común, decide emplear la regla del VMC; es decir, desde su ciudad de origen visitará aquella otra ciudad inmediatamente más cercana y así sucesivamente. Cuando don Pablo ha recorrido el $75 \%$ de las ciudades programadas, aún le queda disponible el 67\% del dinero que destinó para sus vacaciones. Todo se comporta según lo esperado, continúa colocando en práctica su estrategia, pero cuando ha visitado 122 ciudades se da cuenta que solo dispone de 101 millones de pesos y dice: ¿cómo es posible?, ¿cómo pude haber gastado 124 millones de pesos visitando solo 25 ciudades, cuando antes visité 97 ciudades con 112 millones de pesos?.

Viene la preocupación de don Pablo, ya que dispone del 30\% de su presupuesto inicial, pero luego de analizar, se tranquiliza al notar que solo le faltan 8 ciudades por visitar. $Y$ dice, ahora más que nunca es prioritario visitar las ciudades inmediatamente

Tabla 2. Fuentes de información para las comparaciones (Adaptada de PÉREZ, 2011a).

\begin{tabular}{|c|c|c|c|}
\hline Fuente de información & Pais de afiliación de autores* & Familia & Métodos** \\
\hline \multirow{2}{*}{ Wang y Wang (2008) } & \multirow{2}{*}{ China } & $\mathrm{CH}$ & MNACO \\
\hline & & $\mathrm{CH}$ & MMAS \\
\hline \multirow{3}{*}{ Velayudhan et al. (2007) } & \multirow{3}{*}{ USA } & $\mathrm{CH}$ & ACO MP \\
\hline & & $\mathrm{CH}$ & ACO SLR \\
\hline & & $\mathrm{CH}$ & ACO MLR \\
\hline \multirow{2}{*}{ Dorigo y Gambardella (1997) } & \multirow{2}{*}{ Bélgica y Suiza } & $\mathrm{CH}$ & ACS \\
\hline & & $\mathrm{AG}$ & $\mathrm{GA}$ \\
\hline \multirow{4}{*}{ Sallabi y El-Haddad (2009) } & \multirow{4}{*}{ Libia } & $\mathrm{AG}$ & FRAG_GA \\
\hline & & $\mathrm{AG}$ & SWAP_GATSP \\
\hline & & $\mathrm{AG}$ & OX_SIM \\
\hline & & $\mathrm{AG}$ & $1 \mathrm{GA}$ \\
\hline Luo, Yang y li (2008) & China & Otros & SFLA \\
\hline Teodorovic (2008) & Serbia & Otros & $\mathrm{BCO}$ \\
\hline \multirow{3}{*}{ Jeong, Kim y Lee (2009) } & \multirow{3}{*}{ Repúbica de Korea } & ES & CSA \\
\hline & & ES & TFSA \\
\hline & & ES & TRFSA \\
\hline \multirow{3}{*}{ Dos Santos (2009) } & \multirow{3}{*}{ Brasil } & $\mathrm{AG}$ & AG serial \\
\hline & & $\mathrm{AG}$ & AG paralelo \\
\hline & & $\mathrm{AG}$ & AG grupo \\
\hline
\end{tabular}

* Se refiere al país donde pertenece la institución a la que, al momento de la publicación, figuraron estar afiliados los autores de cada artículo; ** Algunos son desarrollos de dichos autores, mientras que otros son citados por ellos y empleados para compararse; CH: Colonia de hormigas; ES: Enfriamiento simulado; AG: Algoritmos genéticos 
cercanas. Siempre funcionó antes, debe seguir funcionado, manifiesta con optimismo. La suerte de don Pablo no está a su favor, a medida que visita las demás ciudades y observa su billetera, nota que su dinero se reduce con mayor impacto. Lleno de preocupaciones, se aferra más a su estrategia del VMC, pero parece que no da resultado. Cuando regresa a casa después de visitar 130 ciudades diferentes, empleando su estrategia del VMC, debe 91 millones de pesos. Las cosas se salieron de las manos. En este instante reflexiona y dice "funcionó antes, por qué no funciona ahora”. Esta historia, cuyos resultados consolidados pueden verse en la Figura 1, ilustra los insumos y hechos necesarios para ahondar en el análisis de la estrategia del VMC desde el pensamiento sistémico.
Aparte de la instancia empleada para ilustrar el caso de don Pablo, este mismo comportamiento prevalece en otras instancias del TSP simétrico, a excepción de aquella idealizada donde todas las ciudades son equidistantes, lo cual no es común encontrar en la realidad. En la Figura 2, por ejemplo, se presentan algunas evidencias del comportamiento del costo acumulado a media que se construyeron tours, mediante el VMC, para instancias simétricas de 169 y 141 ciudades.

\subsection{El caso de Don Pablo visto desde algunas leyes del pensamiento sistémico}

"Los problemas de hoy derivan de las soluciones de ayer" (SENGE, 1998, p.77-78): Peter Senge inicia

Costo acumulado a medida que el viajero recorre las 130 ciudades bajo VMC

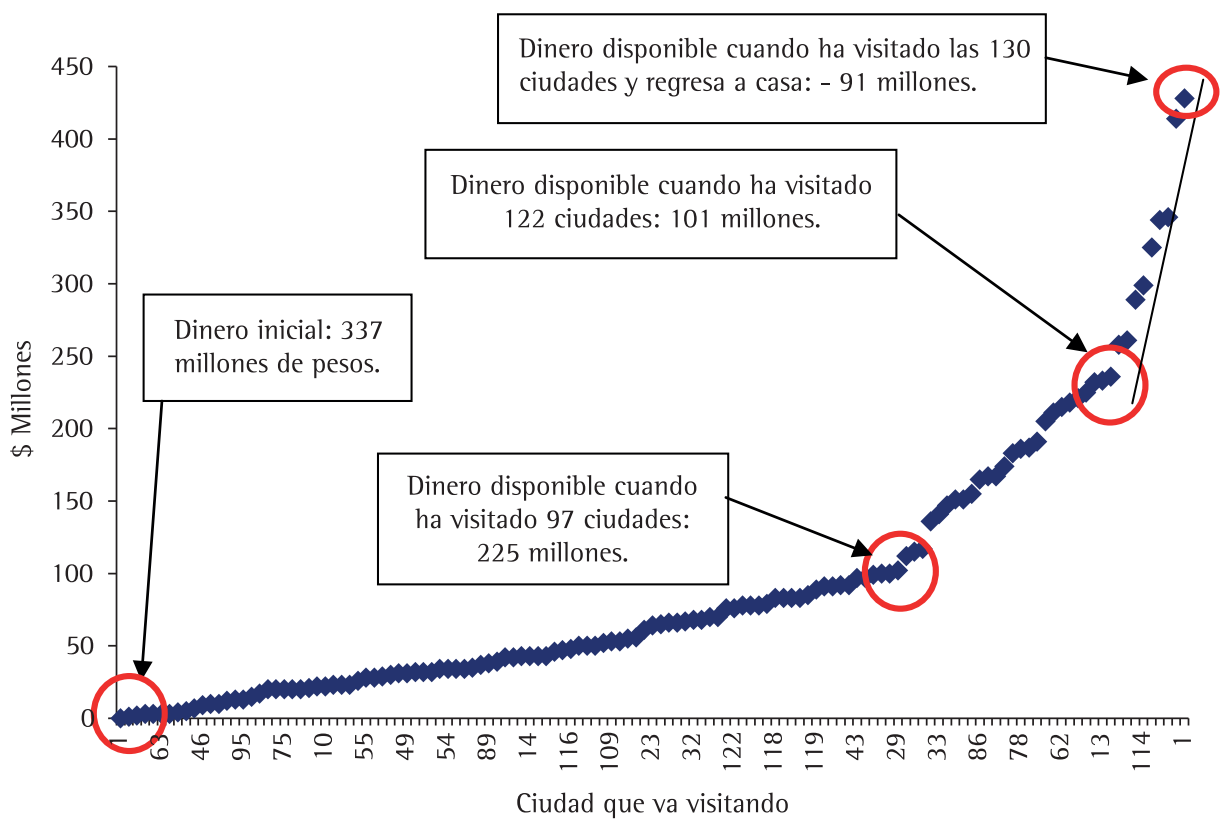

Figura 1. Comportamiento del costo acumulado hasta que visitó las 130 ciudades bajo el VMC. Respuesta mediante el WinQSB y procesando los datos en Excel (PÉREZ, 2011a).

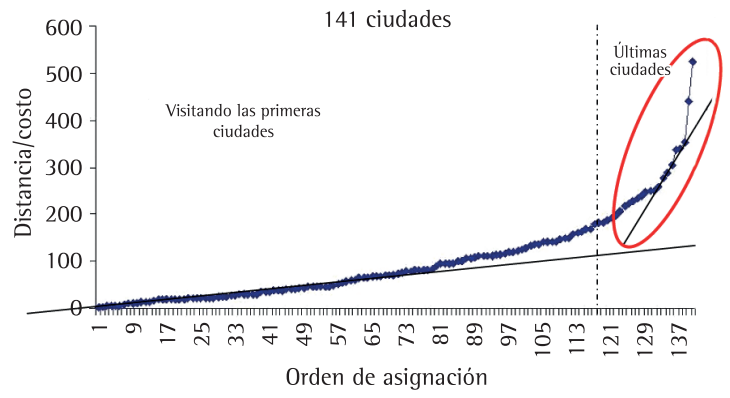

(a)

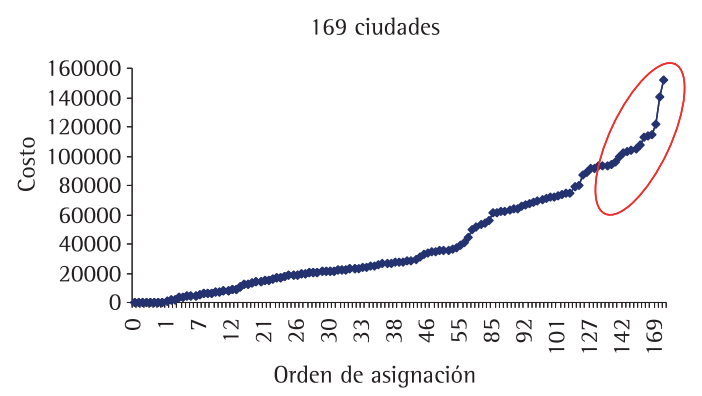

(b)

Figura 2. Costo acumulado para TSP de 169 y 141 ciudades bajo el VMC. (adaptado de PÉREZ, 2011a). 
esta primera ley de la quinta disciplina refiriéndose a que "a menudo nos desconcierta la causa de nuestros problemas, cuando solo necesitamos examinar nuestras propias soluciones a otros problemas en el pasado". Antes de que don Pablo ejecutara el plan vacacional de 130 ciudades, el problema, enfocado en realizar un tour que le saliera lo más económico posible, le buscó una aparente solución inicial. Cada que llegaba a una ciudad disfrutaba de ella y luego se desplazaba hacia la vecina más cercana y, así sucesivamente, cada que se enfrentaba a la pregunta ¿qué ciudad visitar?, empleaba aquella solución que le ha venido funcionando. No obstante, como pudo verse, la práctica continua de esta solución se ha constituido en la causa del problema que hoy don Pablo enfrenta, y es precisamente todo lo contrario a lo que deseaba lograr. Luego de que ha visitado las 130 ciudades, es más, generando sospecha desde que visitó 97 ciudades, ha concluido que aquella solución que funcionó antes, fue contraproducente, llevándolo a deber en la actualidad 91 millones de pesos.

“Cuanto más se presiona, más presiona el sistema" (SENGE, 1998, p. 78-80): Peter Senge la ilustra con ejemplos como: "cuanto más trabajaba, más trabajo había”. En el caso de don Pablo, cuando el dinero se iba consumiendo de manera más acelerada, más necesidad sentía don Pablo de tomar acciones drásticas para mitigar el aparente consumo exagerado, y se veía tentado a visitar ciudades inmediatamente cercanas disponibles. El presionaba cada vez con más fuerza después de haber visitado 97 ciudades, pero el sistema presionaba aún más con la misma consecuencia.

"La causa y el efecto no están próximos en el tiempo y en el espacio" (SENGE, 1998, p. 84-85): Los síntomas de agotamiento del dinero disponible, fruto de la acción de ejecutar el VMC desde el inicio, no pudieron notarse sino hasta que don Pablo llegó a la ciudad 98 en adelante. La acción ocurrió en la primera ciudad que visitó, pero ese efecto dominó, sólo comenzó a ser visible luego de haber disfrutado de sus vacaciones en 97 ciudades, siendo aún más notorio el efecto al tener que regresar a su ciudad de origen. Don Pablo obvió ello, simplemente se preocupó por probar la acción del VMC desde una ciudad y evaluó sus resultados $\mathrm{y}$, al notar que iba bien, concluyó que el efecto global que estaba generando era satisfactorio, ya que no consideró esta ley del pensamiento sistémico.

Al caso de don Pablo también es viable encontrarle otras leyes como "la cura puede ser peor que la enfermedad" y "El camino fácil lleva al mismo lugar". Para profundizar en estos análisis remítase a Pérez (2011a).

\subsubsection{Arquetipo sistémico que subyace en el caso de Don Pablo}

En el caso de don Pablo subyace el arquetipo sistémico expuesto en la Figura 3.

Este arquetipo es similar al que Senge (1998) llama "soluciones contraproducentes". Considere la siguiente interpretación: don Pablo, en su modelo mental, a medida que visita cada ciudad espera tener una cantidad de dinero disponible. Durante las primeras visitas y hasta pasear por 97 ciudades, observa el estado de la brecha entre lo que el espera y lo que realmente está obteniendo $\mathrm{y}$, como esta brecha en los primeros instantes de sus vacaciones se asemeja a cero, entonces siente cada vez más necesidad de mantener casi exactos lo deseado y lo real, en términos del dinero disponible. Esto lo conduce a seguir empleando su estrategia estrella "vecino más cercano" y continúa el ciclo compensador (balanza), teniendo la sensación de que todo está controlado y que será inevitable que logre cumplir su ciclo vacacional con el dinero disponible. Lo descrito hace referencia a un lapso de tiempo que se llamará momento 1 y abarca desde que inicia el ciclo vacacional hasta que ha visitado 97 ciudades. Viene ahora el momento 2: instante en que comienza a vacacionar en las demás ciudades. Allí, la ejecución constante de la estrategia del VMC por parte de don Pablo, ha llevado a que la disponibilidad de ciudades inmediatamente cercanas a visitar comience a agotarse, por lo cual no tendrá más remedio que desplazarse hacia la siguiente más cercana, que aún no ha visitado; no obstante, ésta puede ubicarse lejos de donde él se encuentra. Ello repercutirá en una mayor brecha entre lo que desea y lo que está observando en la realidad. Preocupado por la situación, cada vez nota que a pesar de sus esfuerzos constantes de "lo más cercano", los buenos resultados no se están viendo. El sistema está respondiendo con más fuerza debido al ciclo de retroalimentación reforzadora (bola de nieve).

De acuerdo con Senge (1998, p. 475), esta estructura contiene una solución eficaz en el corto plazo, que en el caso de don Pablo se asocia al momento 1 , pero que tiene consecuencias imprevistas a largo plazo (momento 2), que generan más necesidad de la misma solución. El principio recomendado para abordar esta estructura es "no descuides el largo plazo. De ser posible no recurras a soluciones de corto plazo, o úsalas solo para ganar tiempo mientras trabajas en un remedio duradero". (SENGE, 1998, p. 475). 


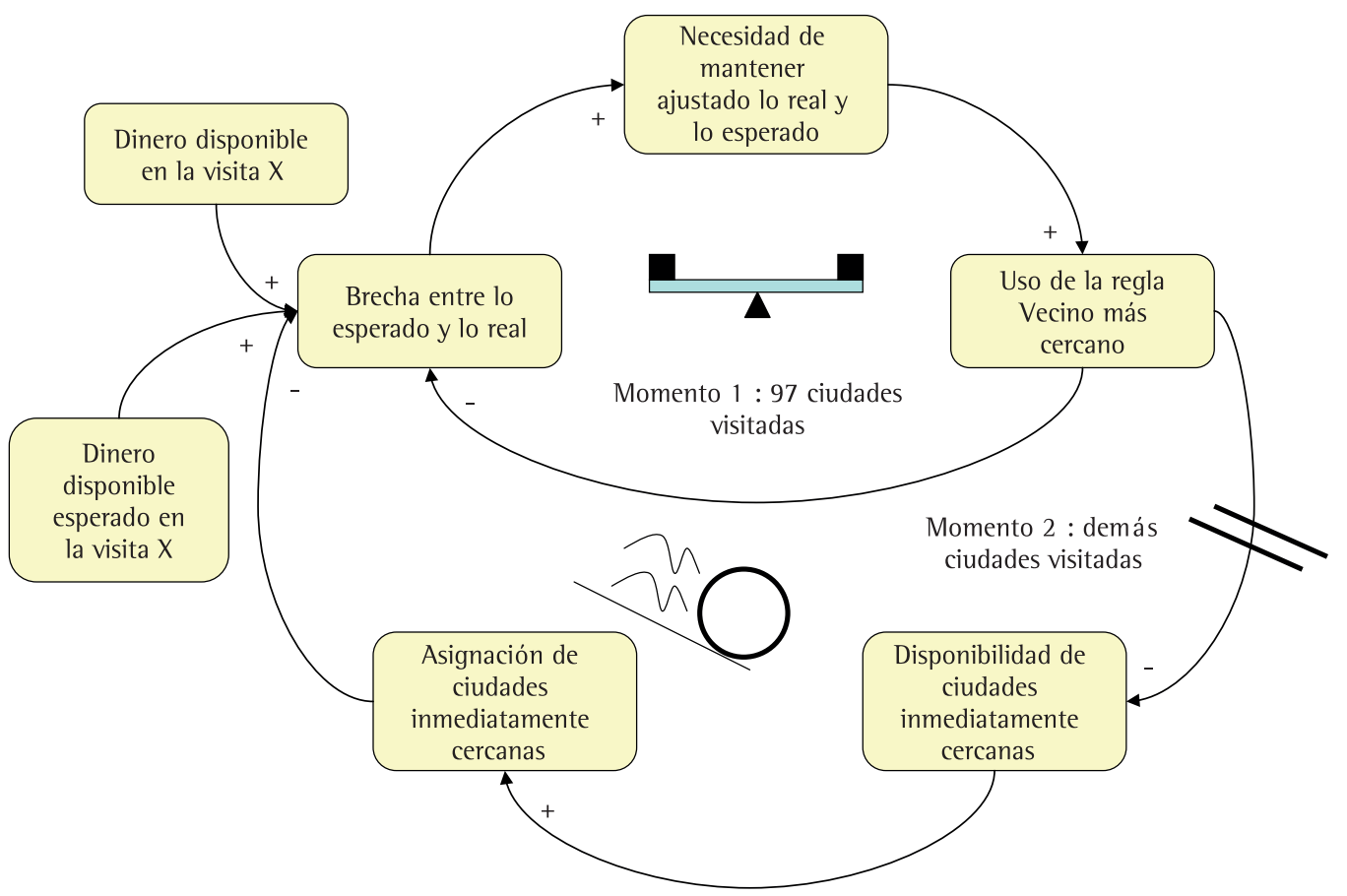

Figura 3. Arquetipo sistémico de la estrategia del VMC ejemplificado para la instancia de 130 ciudades. (PÉREZ, 2011a).

\subsection{Método heurístico a proponer}

\subsubsection{Diseño de la heurística}

El diseño del método a proponer parte de la búsqueda de una estrategia de mejoramiento que permita intervenir la estructura sistémica identificada para la regla de decisión vecino más cercano. En ese sentido, al recurrir de nuevo a otras leyes del pensamiento sistémico, una de ellas manifiesta que “[...] los cambios pequeños pueden producir resultados grandes, pero las zonas de mayor apalancamiento a menudo no son obvias [...]" (SENGE, 1998, p. 85-86).

Así, entonces, según Senge (1998), el pensamiento sistémico señala que los pequeños cambios, focalizados en zonas apropiadas, en casos producen mejoras significativas y duraderas.

Lo anterior, sumado a estrategias propias del diseño de heurísticas, como son: creatividad, imaginación, entre otros, han dado lugar a una modificación que se desea probar en el sistema: plan vacacional usando VMC. Esta alteración se enfoca en que el viajero (don Pablo), cuando se encuentre en una ciudad determinada decida renunciar a trasladarse hacia la inmediatamente cercana, pasando a otra, a fin de que posteriormente, ese aparente sacrificio lo ayude a hacer mejor uso del dinero disponible, quizá regresando al punto de inicio desde una ciudad menos lejana. Esta nueva regla se denominará Sacrificio cortoplacista. Pero allí surgen dos interrogantes ¿de qué magnitud debe ser el sacrificio? y ¿en qué ciudad realizarse? Teniendo presente que el cambio debe ser leve $y$, a su vez, producir un efecto importante en el resultado final del sistema, este estudio ahondará esfuerzos en la renuncia a la ciudad inmediatamente cercana, para pasar a la segunda ciudad inmediatamente cercana, disponible. Así, este Sacrificio cortoplacista a efectuar se conocerá como de nivel 2 , siendo el de nivel 1 el mismo VMC, puesto que visita la primera ciudad más cercana disponible. De nivel 3 equivale a renunciar para visitar la tercera ciudad más cercana disponible y así sucesivamente. El posible punto de apalancamiento, incorporando en un momento determinado la regla Sacrificio cortoplacista pretende estabilizar la estructura "bola de nieve" (momento 2), como se muestra en la Figura 4.

Con el Sacrificio cortoplacista se espera que posteriormente (líneas cortas de retardo temporal) haya más disponibilidad de ciudades inmediatamente cercanas que sirvan para reducir la brecha entre la expectativa de dinero disponible y lo real. Luego de un sacrificio cortoplacista sigue de nuevo el VMC, posibilitando que el sistema se adapte a su estrategia original. Así, el Sacrificio cortoplacista junto con la adaptación de nuevo a la práctica del VMC, se denominará Sacrificio Cortoplacista Adaptativo (SCA). Con el SCA se busca una posible estabilización del 
impacto de la estrategia del VMC en las últimas ciudades del ciclo vacacional. Esto lo refleja el ciclo compensador que subyace al considerar las relaciones más externas, líneas punteadas (Figura 4). Para una mejor ilustración, a nivel de las iteraciones que representa el Sacrificio cortoplacista adaptativo, y partiendo de una instancia TSP de cinco ciudades, considere el ejemplo expuesto en la Figura 5.
En la Figura 5, se parte de la solución arrojada por el VMC, luego se realiza el primer sacrificio cortoplacista: se renuncia a la ciudad 5 (inmediatamente cercana desde la 1) para visitar la segunda más cercana disponible (ciudad 3) y, a partir de la ciudad 3, se aplica de nuevo la regla del VMC. Este nuevo tour (1-3-2-4-5-1) dio como resultado el mismo costo del VMC (8). Para generar el siguiente tour, se regresa al

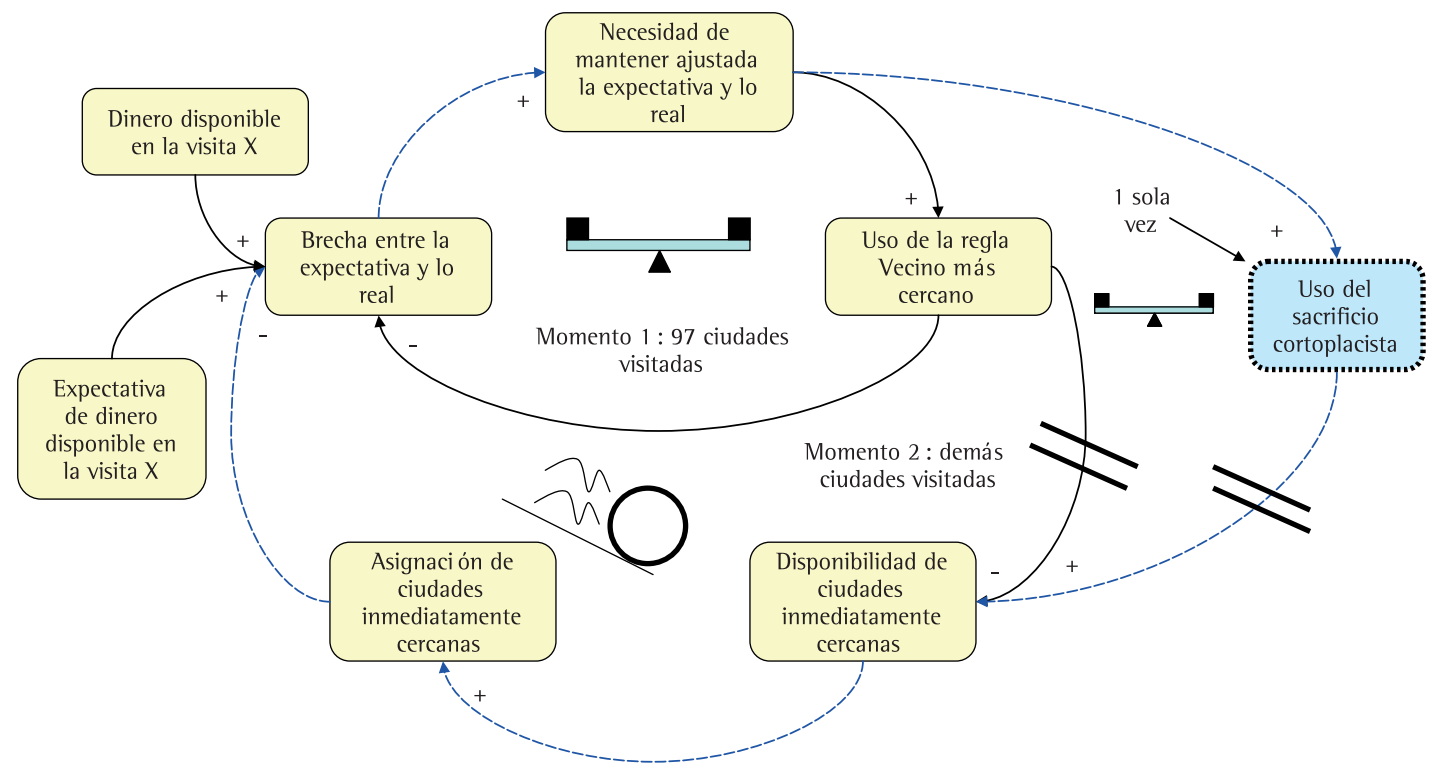

Figura 4. Propuesta estructural, sistémica, para mejorar el desempeño que subyace en la estrategia del VMC (PÉREZ, 2011a).

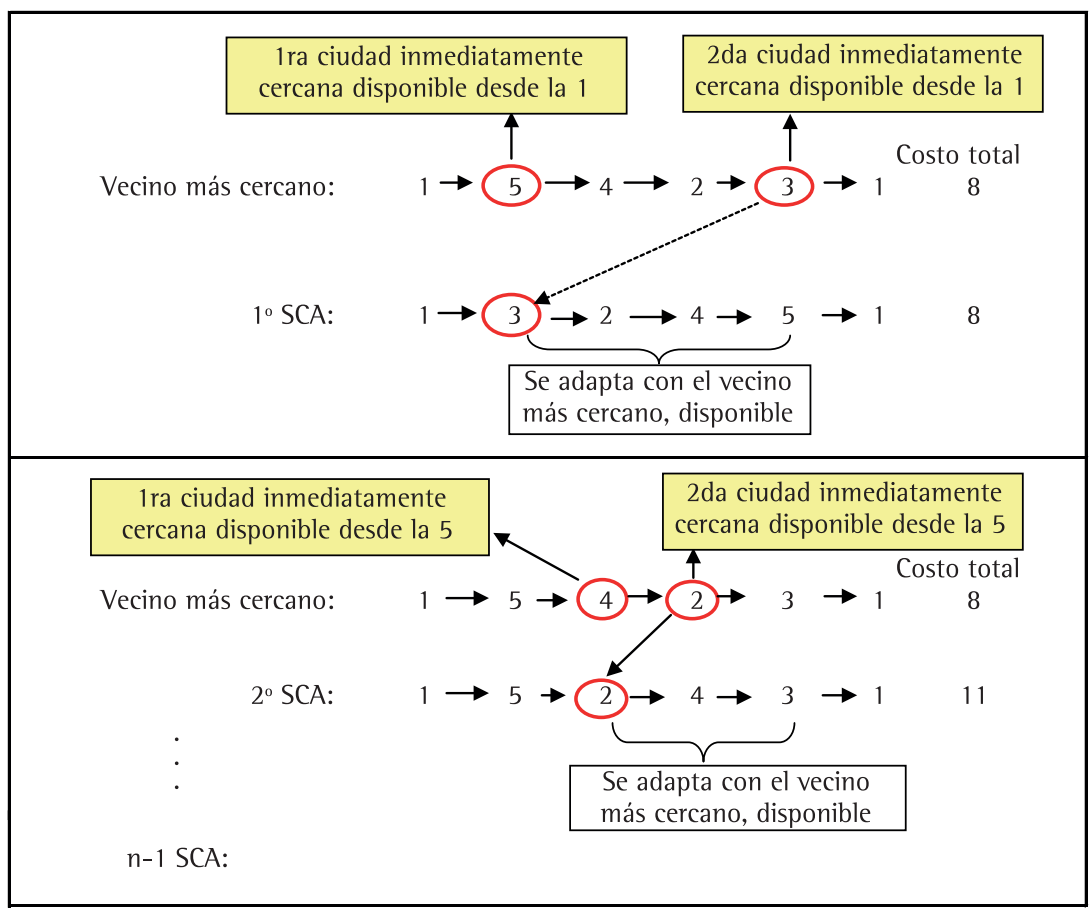

Figura 5. llustración del SCA para una instancia TSP de cinco ciudades (PÉREZ, 2011a). 
construido bajo el VMC, a fin de garantizar que la búsqueda sea global. Apoyados en Berbiela (1998) y aplicado al presente contexto, este tipo de búsqueda significa que, antes de generar el nuevo el tour, se borra de la memoria cualquier operación previa hecha en el sistema, a fin de buscar y generar otra nueva mejor solución. Así, se dejan fijas las ciudades 1 y 5 , y se realiza el sacrificio desde la ciudad 5. No se visitará la primera más cercana disponible, sino la siguiente (2). A partir de la ciudad 2 se aplica de nuevo la regla del VMC y así sucesivamente. Al final, si $n$ es el tamaño de la instancia TSP, en este caso 5 ciudades, se realizarán n-1 sacrificios cortoplacistas adaptativos. Es decir, con esta heurística se tendrán n-1 nuevos tours por analizar.

Nótese el pequeño cambio que se está incorporando al comportamiento del sistema vacacional bajo el VMC. Esta leve modificación busca que permita encontrar en qué ciudad debería realizarse el sacrificio cortoplacista adaptativo, con el ánimo de que quizá mejore el desempeño final del sistema ilustrado. Observe que antes de iniciar cada SCA, se retoma la solución de inicio arrojada por el VMC. Esto, desde el punto de vista de la optimización combinatoria, y como se mencionó, constituye una búsqueda global, lo cual es pertinente para evitar el encierro en óptimos locales. No obstante, aparte de considerar, en el diseño, diversos principios del pensamiento sistémico como campo de inspiración, no conviene dejar de lado las recomendaciones fundamentales arrojadas por la revisión literaria del TSP (PÉREZ, 2011a). La primera fue que para mover las puntas actuales de conocimiento hacia esferas desconocidas, es preciso incorporar nuevos campos de inspiración, lo cual ya se atendió. La otra arrojó que, para avanzar alrededor del conocimiento actual es preciso generar híbridos de los métodos existentes, presentándose una tendencia a combinar búsqueda global con la local. Para vincular esta última recomendación, cada tour generado con la leve modificación, fruto de cada SCA, será alterado mediante una búsqueda local. Para ello, la revisión literaria (PÉREZ, 2011a) mostró como principales optimizadores locales el 2-opt y el 3-opt.

Al tratarse de un primer estudio sobre la pertinencia del pensamiento sistémico como posible campo de inspiración para el desarrollo de métodos de optimización combinatoria, no se probará con $\mathrm{k}$ superiores a 2, puesto que Golden et al. (1980) y Marti (2003) concluyeron que para ello el aumento en el tiempo computacional no justifica la eficacia en la respuesta. La adaptación que se incorpora en el sacrificio cortoplacista (sección 3.2.1) junto con la búsqueda local 2opt, buscan emular el principio de la homeostasis de los sistemas: "su capacidad para mantener las condiciones de supervivencia en un ámbito cambiante" (SENGE, 1998).

\subsubsection{Programación de la heurística}

Diagrama de flujo: En la Figura 6 se presenta la estructura general, en forma de diagrama de flujo, que representa el algoritmo Sacrificio Cortoplacista Adaptativo con 2opt (SCA_2opt).

La programación del algoritmo fue realizada en Visual Basic bajo Macros de Excel.

\subsubsection{Validación de la heurística propuesta mediante su uso en el caso de don Pablo}

Recuérdese que con la heurística propuesta se pretende estabilizar el comportamiento de "bola de nieve" que subyace en el caso de don Pablo en las últimas ciudades que visita (Figura 4). Al aplicar el SCA_2opt en la instancia de 130 ciudades, en la Figura 7, lado derecho, se nota al principio un comportamiento similar al realizado con el VMC (lado izquierdo), luego una ruptura (sacrificio) y después una adaptación (VMC y 2opt). Así, entonces, ante el uso del VMC en la instancia TSP del caso de don Pablo se obtiene un costo total de 428 millones de pesos, en tanto que bajo el SCA_2opt dicho costo se reduce a 298 millones de pesos.

\section{4. ¿El desempeño del SCA_2opt es resultado del análisis sistémico?}

Surge el deseo de estudiar si el desempeño del método propuesto realmente se debe al posible punto de apalancamiento que se le hizo a la heurística VMC, o por el contrario, es fruto del potencial de la búsqueda local 2_opt que le fue incorporada, o hasta quizá del azar. En otras palabras, ¿el desempeño se debe al uso del pensamiento sistémico como campo de inspiración? Así, entonces, si el desempeño de la heurística SCA_2opt se debe al análisis sistémico efectuado, el hecho de eliminar el SCA e inicializar exactamente la misma búsqueda local 2opt con la solución del VMC o con un tour generado aleatoriamente, debería dar un desempeño menos favorable. En ese sentido, se probó el desempeño de dos heurísticas más: VMC_2opt y Aleat_2opt, cuyas estructuras, partiendo de la empleada por el SCA_2opt, se ilustran en la Figura 8.

Analizando la eficacia de los métodos, al efectuar ANOVA para estas tres heurísticas, probando con instancias que van desde 80 hasta 140 ciudades (dos diferentes de cada tamaño y con incrementos de 10), se encontró un valor $p$ nulo para el factor método $(0,0000)$. Es decir, al menos una de las heurísticas sometidas a prueba presenta un costo diferente respecto a las demás. Pero ¿cuál o cuáles 
"Inicia con el sacrificio cortoplacista adaptativo en la ciudad ubicada en la posición t. Siendo N el número de ciudades a visitar

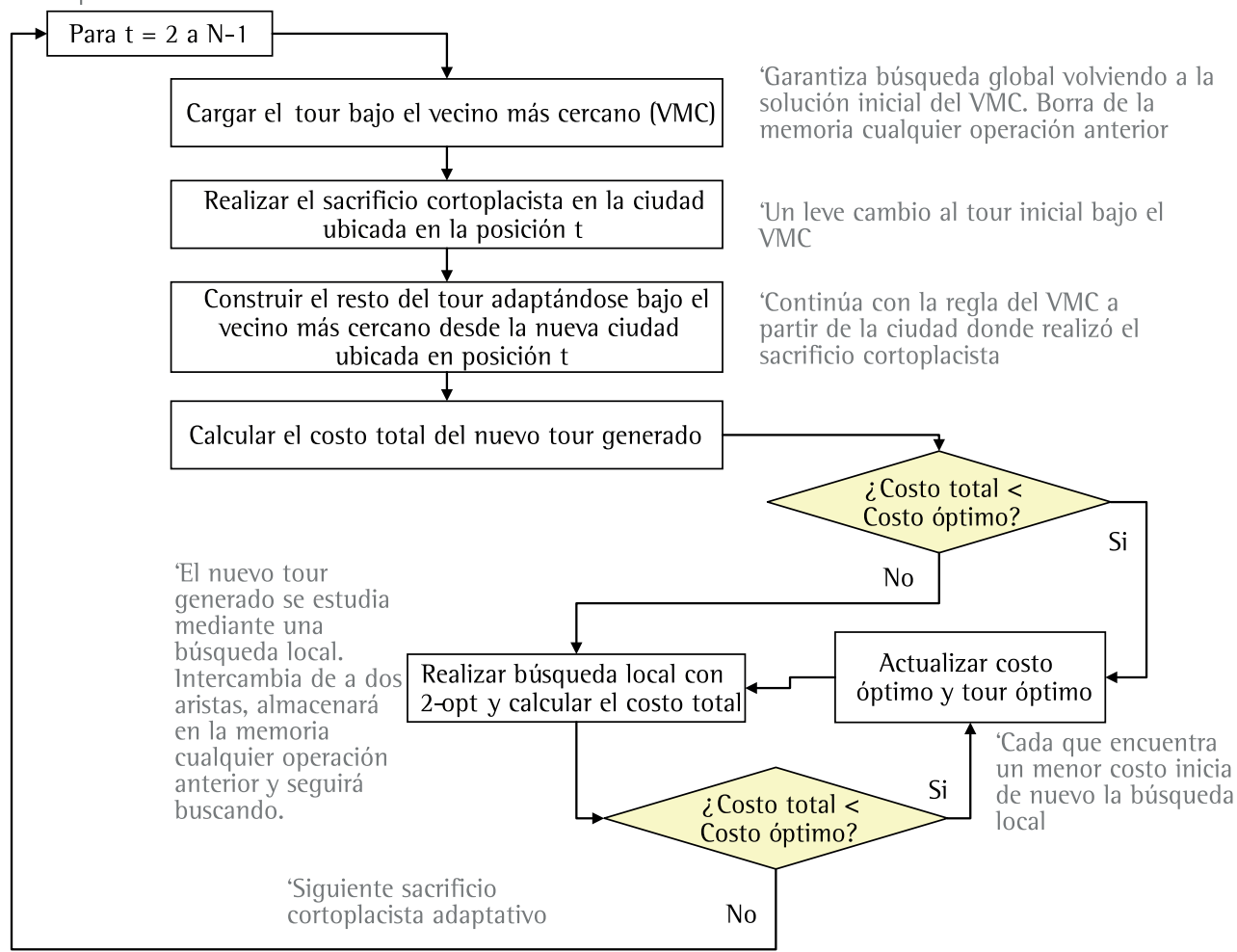

Figura 6. Diagrama de flujo del SCA_2opt. Fuente (PÉREZ, 2011a).
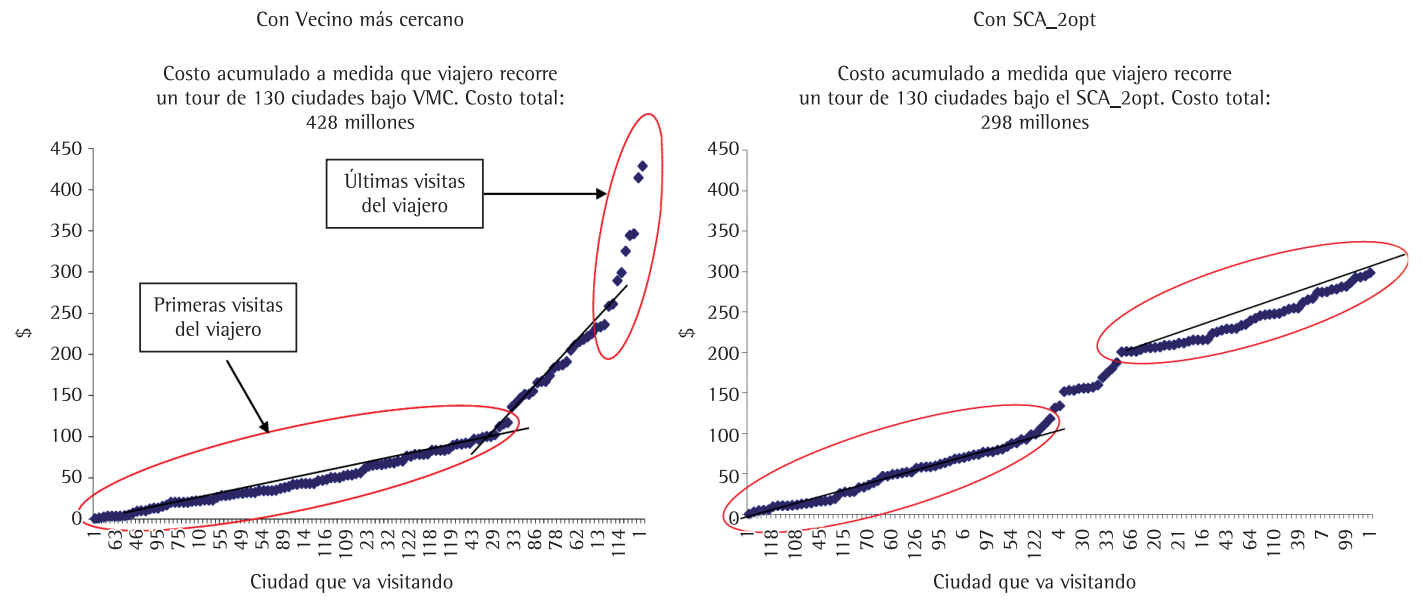

Figura 7. Costo total acumulado a medida que se construye el tour de 130 ciudades del caso de don Pablo, bajo el VMC y el SCA_2opt. (Adaptada de PÉREZ, 2011a).

de ellas se diferenciarán? La prueba de rangos múltiples de Duncan arrojó que estas tres heurísticas presentan, entre sí, diferencias estadísticamente significativas respecto al costo del tour arrojado, siendo el SCA_2opt aquella que menor costo medio presenta $(298,29)$, seguida de VMC_2opt $(350,29)$ y por último Aleat_2opt $(450,5)$. Así, se encuentra que la eficacia del SCA_2opt se debe a la incorporación del punto de apalancamiento, resultado del análisis sistémico del VMC. 
SCA_2opt

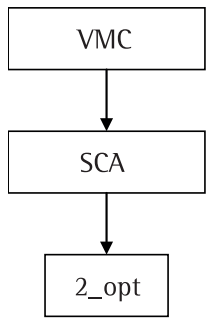

Figura 8. Dos heurísticas alternas para analizar el efecto del SCA.

\subsection{SCA_2opt_r}

El Dmax es un parámetro del 2-opt que representa la mejora permisible para que valga la pena efectuar el intercambio de un par de aristas. En el 2-opt puro, este parámetro se inicializa en cero (0). Por ejemplo, considere el siguiente mejor tour generado en un momento determinado para una instancia TSP de 5 ciudades: 1-3-2-5-4-1. Suponga que se desea analizar la pertinencia del intercambio 2-opt entre las aristas $(3,2)$ y $(5,4)$. Bajo el 2-opt, dicho intercambio sería así: $(3,5)$ y $(2,4)$. Si C representa el costo de una ruta determinada, el mencionado intercambio tendría sentido siempre y cuando (C $(3,5)+C(2,4))-(C(3,2)+C(5,4))<$ Dmax. En otras palabras, sólo valdría la pena el intercambio cuando el primer término $((C)(3,5)+C(2,4))$ de la resta sea menor que el segundo $(C(3,2)+C(5$, 4)). No obstante, como en la heurística a proponer el 2-opt está incorporado dentro de cada sacrificio cortoplacista adaptativo; es decir, se efectúa para cada tour, no en todos los casos conviene inicializar el Dmax en cero, debido a que conduciría a efectuar intercambios para tours de costo menor que el mejor valor encontrado en un determinado SCA, pero no necesariamente respecto al óptimo guardado hasta ese momento. Por ello, en la heurística diseñada, el Dmax se inicializa en cero siempre y cuando cada tour, fruto del SCA mejore la situación global; en caso contrario, el valor inicial es el costo óptimo alojado hasta ese momento (costo_opt) menos el costo total que genera el tour actual (costo_total). Esto evita que se generen intercambios para tours que distan considerablemente del mejor costo encontrado hasta ese entonces; de no hacerlo, llevaría a pérdida de eficiencia computacional.

A pesar de lo dicho, es posible que el costo total arrojado por un tour determinado luego del SCA no sea mejor que el alojado hasta ese entonces, pero que al efectuarle intercambios se llegue al final a un mejor resultado. Para estudiar ello, el Dmax fue igualado a $(1+r) \times$ costo_opt - costo_total, siendo $r$
$[0,1]$ la proporción adicional que se le cargará al mejor costo almacenado hasta ese entonces (costo_opt), de modo que sea más permisible la decisión de efectuar los intercambios. Dado que el algoritmo propuesto permite explorar diversos valores del parámetro, éstos fueron tanteados bajo ensayo y error para observar el desempeño de la heurística SCA_2opt. La optimización del parámetro $r$ representa de por sí un proyecto retador, que se constituye en uno de los trabajos futuros.

Ante la posibilidad de modificar el parámetro $r$, cuando éste sea igual a cero las siglas que representan el método propuesto serán las mismas: SCA_2opt $y$, cuando $r \neq 0$ se tratará como SCA_2opt_r; esto último generó la idea de comparar, en términos de eficacia, el SCA_2opt y el SCA_2opt_r. Para ello, los dos métodos propuestos se ejecutaron en siete instancias de TSPlib (REINELT, 1991), considerándose como variable respuesta la distancia relativa entre el costo arrojado por cada método y el mejor costo reportado en la literatura. En la Tabla 3 se presentan los resultados de las experimentaciones.

Con relación a la distancia relativa del costo arrojado por cada método respecto al mejor valor reportado en la literatura, el menor fue obtenido mediante el SCA_2opt_r, con una media de 2,27\% (considerando todos los valores de la columna A); en tanto que para el SCA_2opt (columna B) este valor se situó en 5,61\%. Respecto a la eficiencia computacional, en promedio, el SCA_2opt_r consumió 14,3\% más de tiempo que el SCA_2opt. Para profundizar al respecto remítase a Pérez (2011a).

\subsection{Comparación contra metaheurísticas bajo análisis multicriterio}

Las heurísticas propuestas pudieron compararse con 19 metaheurísticas, pero considerando grupos de instancias donde estos métodos presentaron información en el binomio eficacia y eficiencia; en un solo grupo la eficacia fue representada por el costo medio, puesto que no se dispuso de información; para los demás, se trabajó con el mejor costo.

Para incorporar el factor eficiencia en el análisis multicriterio se recurrió al tiempo medio (TM) en lugar de gigaciclos de PC (que brinda un conversión aceptable, más no exacta, a unidades equivalentes). Esta elección fue motivada inicialmente como una posible salida, exploratoria, ante la ausencia de información sobre los todos PC en los que se probaron las metaheurísticas tomadas de referentes (PÉREZ, 2011a), pero luego, ello fue reforzado con el hecho de que los resultados de eficiencia empleando la variable gigaciclos de PC favorecieron más al SCA_2opt y al 
SCA_2opt_r, que si se considera el TM, siendo este último, por lo mismo, un escenario más retador en las comparaciones multicriterio a realizar ("peor caso").

En vista de que los factores considerados presentan diferentes unidades de medición (costos y tiempo), fue necesario normalizar los valores. Esto permite representarlos como proporciones, bajo un rango entre 0 y 1 . La normalización fue realizada empleando la Ecuación 1.

$$
D N_{i j k}=\frac{D_{i j k}}{\sum_{i=1}^{5} D_{i j k}} \quad \forall j k
$$

Tabla 3. Resultados de probar los métodos propuestos en siete instancias de TSPlib.

\begin{tabular}{lcccccc}
\hline $\begin{array}{c}\text { Instancias } \\
\text { TSP }\end{array}$ & $\begin{array}{c}\text { (1) Mejor } \\
\text { costo } \\
\text { reportado }\end{array}$ & $\begin{array}{c}\text { Valor } \\
\text { de } r\end{array}$ & $\begin{array}{c}\text { (2) Costo bajo el } \\
\text { SCA_2opt_r }\end{array}$ & $\begin{array}{c}\text { (3) Costo bajo } \\
\text { el SCA_2opt }\end{array}$ & $\begin{array}{c}\text { A. Distancia relativa de (2) } \\
\text { respecto a (1): } \\
100 \% \times[(2)-(1)] /(1)\end{array}$ & $\begin{array}{c}\text { B. Distancia relativa } \\
\text { de (3) respecto a (1): } \\
100 \% \times[(3)-(1)] /(1)\end{array}$ \\
\hline Swiss42 & 1.273 & 0,05 & 1.285 & 1.375 & $0,9 \%$ & $8,0 \%$ \\
Eil51 & 426 & 0,10 & 436,08 & 449,85 & $2,4 \%$ & $5,6 \%$ \\
St70 & 675 & 0,14 & 690,62 & 713,98 & $2,3 \%$ & $5,8 \%$ \\
Eil50 & 425 & 0,10 & 430,48 & 448,83 & $1,3 \%$ & $5,6 \%$ \\
Krob150 & 26.130 & 0,18 & $26.904,48$ & $28.136,3$ & $3,0 \%$ & $2,7 \%$ \\
d198 & 15.780 & 0,01 & $16.180,53$ & 16.212 & $2,5 \%$ & $3,9 \%$ \\
Tsp225 & 3916 & 0,01 & $4.054,77$ & 4.071 & $3,5 \%$ & \\
\hline
\end{tabular}

Tabla 4. Matriz de pagos original y normalizada para la comparación con metaheurísticas (Adaptada de PÉREZ, 2011a).

\begin{tabular}{|c|c|c|c|c|c|c|c|c|c|}
\hline $\begin{array}{l}\text { Instancia; } \\
\text { (1)Eficacia; } \\
\text { (2)Eficiencia }\end{array}$ & $\begin{array}{c}\text { (1) } \\
\text { Eficacia }\end{array}$ & $\begin{array}{c}\text { (2) } \\
\text { Eficiencia }\end{array}$ & Fuente de información & Familia & Métodos & (1) & (2) & $\begin{array}{c}\text { (1) } \\
\text { Norm. }\end{array}$ & $\begin{array}{c}(2) \\
\text { Norm. }\end{array}$ \\
\hline \multirow{7}{*}{$\begin{array}{l}\text { KroA100; (1)MC; } \\
\text { (2)TM }\end{array}$} & \multirow{7}{*}{$\begin{array}{l}\text { Mejor } \\
\text { costo }\end{array}$} & \multirow{7}{*}{$\begin{array}{l}\text { Tiempo } \\
\text { medio }\end{array}$} & \multirow{2}{*}{ Pérez (2011a) } & PSis & SCA_2opt_r & $22.219,34$ & 1,07 & 0,1385 & 0,0034 \\
\hline & & & & PSis & SCA_2opt & $22.379,4$ & 0,87 & 0,1395 & 0,0028 \\
\hline & & & \multirow{2}{*}{ Wang y Wang (2008) } & $\mathrm{CH}$ & MNACO & $21.285,40$ & 26,88 & 0,1327 & 0,0863 \\
\hline & & & & $\mathrm{CH}$ & MMAS & $21.285,40$ & 104,8 & 0,1327 & 0,3363 \\
\hline & & & \multirow{3}{*}{$\begin{array}{l}\text { Velayudhan et al. } \\
\text { (2007) }\end{array}$} & $\mathrm{CH}$ & ACO MP & 25.483 & 26 & 0,1589 & 0,0834 \\
\hline & & & & $\mathrm{CH}$ & ACO SLR & $24.229,9$ & 22 & 0,1510 & 0,0706 \\
\hline & & & & $\mathrm{CH}$ & ACO MLR & $23.531,40$ & 130 & 0,1467 & 0,4172 \\
\hline \multirow{11}{*}{$\begin{array}{l}\text { KroA100; (1)MC; } \\
\text { (2)NT }\end{array}$} & \multirow{11}{*}{$\begin{array}{l}\text { Mejor } \\
\text { costo }\end{array}$} & \multirow{11}{*}{$\begin{array}{l}\text { Número } \\
\text { de tours }\end{array}$} & \multirow{2}{*}{ Pérez (2011a) } & PSis & SCA_2opt_r & $22.219,3$ & 206 & 0,0898 & 0,0015 \\
\hline & & & & PSis & SCA_2opt & $22.379,4$ & 140 & 0,0905 & 0,0010 \\
\hline & & & \multirow{2}{*}{$\begin{array}{l}\text { Dorigo y Gambardella } \\
\text { (1997) }\end{array}$} & $\mathrm{CH}$ & ACS & 21.282 & 4.820 & 0,0860 & 0,0343 \\
\hline & & & & $\mathrm{AG}$ & $\mathrm{GA}$ & 21.761 & 103.000 & 0,0880 & 0,7325 \\
\hline & & & \multirow{3}{*}{$\begin{array}{l}\text { Velayudhan et al. } \\
\text { (2007) }\end{array}$} & $\mathrm{CH}$ & ACO MP & 25.483 & 400 & 0,1030 & 0,0028 \\
\hline & & & & $\mathrm{CH}$ & ACO SLR & $24.229,9$ & 400 & 0,0980 & 0,0028 \\
\hline & & & & $\mathrm{CH}$ & ACO MLR & $23.531,4$ & 400 & 0,0951 & 0,0028 \\
\hline & & & \multirow{4}{*}{$\begin{array}{l}\text { Sallabi y El-Haddad } \\
\text { (2009) }\end{array}$} & AG & FRAG_GA & 21.282 & 800 & 0,0860 & 0,0057 \\
\hline & & & & AG & SWAP_GATSP & 21.504 & 5.000 & 0,0869 & 0,0356 \\
\hline & & & & AG & OX_SIM & 22.400 & 25.000 & 0,0906 & 0,1778 \\
\hline & & & & AG & $1 \mathrm{GA}$ & 21.282 & 454 & 0,0860 & 0,0032 \\
\hline \multirow{6}{*}{$\begin{array}{l}\text { Eil51; (1)MC; } \\
\text { (2)TM }\end{array}$} & \multirow{6}{*}{$\begin{array}{l}\text { Mejor } \\
\text { costo }\end{array}$} & \multirow{6}{*}{$\begin{array}{l}\text { Tiempo } \\
\text { medio }\end{array}$} & \multirow{2}{*}{ Pérez (2011a) } & PSis & SCA_2opt_r & 436,08 & 0,219 & 0,1676 & 0,0022 \\
\hline & & & & PSis & SCA_2opt & 449,85 & 0,109 & 0,1729 & 0,0011 \\
\hline & & & \multirow{2}{*}{ Wang y Wang (2008) } & $\mathrm{CH}$ & MNACO & 428,87 & 10,98 & 0,1649 & 0,1124 \\
\hline & & & & $\mathrm{CH}$ & MMAS & 428,98 & 40 & 0,1649 & 0,4093 \\
\hline & & & Luo, Yang y li (2008) & Otros & SFLA & 428,87 & 17,42 & 0,1649 & 0,1782 \\
\hline & & & Teodorovic (2008) & Otros & $\mathrm{BCO}$ & 428,87 & 29 & 0,1649 & 0,2967 \\
\hline \multirow{5}{*}{$\begin{array}{l}\text { Eil76; (1)MC; } \\
\text { (2)NT }\end{array}$} & \multirow{5}{*}{$\begin{array}{l}\text { Mejor } \\
\text { costo }\end{array}$} & \multirow{5}{*}{$\begin{array}{l}\text { Número } \\
\text { de tours }\end{array}$} & \multirow{2}{*}{ Pérez (2011a) } & PSis & SCA_2opt_r & 559,61 & 964 & 0,1889 & 0,0019 \\
\hline & & & & PSis & SCA_2opt & 567,01 & 118 & 0,1914 & 0,0002 \\
\hline & & & \multirow{3}{*}{$\begin{array}{l}\text { Jeong, Kim y Lee } \\
\text { (2009) }\end{array}$} & ES & CSA & 613,70 & 204.000 & 0,2072 & 0,3988 \\
\hline & & & & ES & TFSA & 603,68 & 159.000 & 0,2038 & 0,3108 \\
\hline & & & & ES & TRFSA & 618,03 & 147.500 & 0,2087 & 0,2883 \\
\hline \multirow{5}{*}{$\begin{array}{l}\text { Eil76; (1)CM; } \\
\text { (2)TM }\end{array}$} & \multirow{5}{*}{$\begin{array}{l}\text { Costo } \\
\text { medio }\end{array}$} & \multirow{5}{*}{$\begin{array}{l}\text { Tiempo } \\
\text { medio }\end{array}$} & \multirow{2}{*}{ Pérez (2011a) } & PSis & SCA_2opt_r & 559,61 & 1,4 & 0,1932 & 0,0005 \\
\hline & & & & PSis & SCA_2opt & 567,01 & 0,352 & 0,1957 & 0,0001 \\
\hline & & & & $\mathrm{AG}$ & AG serial & 591 & 1.390 & 0,2040 & 0,4741 \\
\hline & & & Dos Santos (2009) & $\mathrm{AG}$ & AG paralelo & 590 & 790 & 0,2037 & 0,2695 \\
\hline & & & & AG & AG grupo & 589 & 750 & 0,2033 & 0,2558 \\
\hline
\end{tabular}


Rave, J. P. et al.

Sacrificio cortoplacista ... pensamiento sistémic. Production, v. 24, n. 1, p. 26-40, jan./mar. 2014

Tabla 5. Desempeño de las métodos ante cinco escenarios (Adaptada de PÉREZ, 2011a).

\begin{tabular}{|c|c|c|c|c|c|c|c|c|c|c|c|c|c|c|}
\hline \multirow{2}{*}{$\begin{array}{l}\text { Instancia; } \\
\text { (1)Eficacia; } \\
\text { (2)Eficiencia }\end{array}$} & \multirow{2}{*}{$\begin{array}{l}\text { País de la } \\
\text { fuente de } \\
\text { información }\end{array}$} & \multirow{2}{*}{$\begin{array}{l}\text { Fuente de } \\
\text { información }\end{array}$} & \multirow{2}{*}{ Familia } & \multirow{2}{*}{ Métodos } & \multicolumn{2}{|c|}{$\begin{array}{l}\text { Eficacia }(100 \%) \\
\text { Eficiencia }(0 \%)\end{array}$} & \multicolumn{2}{|c|}{$\begin{array}{c}\text { Eficacia }(80 \%) \\
\text { Eficiencia }(20 \%)\end{array}$} & \multicolumn{2}{|c|}{$\begin{array}{c}\text { Eficacia (50\%) } \\
\text { Eficiencia }(50 \%)\end{array}$} & \multicolumn{2}{|c|}{$\begin{array}{c}\text { Eficacia }(20 \%) \\
\text { Eficiencia }(80 \%)\end{array}$} & \multicolumn{2}{|c|}{$\begin{array}{c}\text { Eficacia }(0 \%) \\
\text { Eficiencia }(100 \%)\end{array}$} \\
\hline & & & & & (DNi) & Puesto & (DNi) & Puesto & (DNi) & Puesto & (DNi) & Puesto & (DNi) & Puesto \\
\hline \multirow{7}{*}{$\begin{array}{l}\text { KroA100; } \\
\text { (1)MC; } \\
\text { (2)TM }\end{array}$} & \multirow{2}{*}{ Colombia } & \multirow{2}{*}{ Pérez (2011a) } & PSis & SCA_2opt_r & 0,1385 & 3 & 0,1115 & 1 & 0,0710 & 1 & 0,0304 & 2 & 0,0034 & 2 \\
\hline & & & PSis & SCA_2opt & 0,1395 & 4 & 0,1122 & 2 & 0,0712 & 2 & 0,0301 & 1 & 0,0028 & 1 \\
\hline & \multirow{2}{*}{ China } & \multirow{2}{*}{$\begin{array}{l}\text { Wang y Wang } \\
\text { (2008) }\end{array}$} & $\mathrm{CH}$ & MNACO & 0,1327 & 1 & 0,1234 & 3 & 0,1095 & 3 & 0,0955 & 4 & 0,0863 & 5 \\
\hline & & & $\mathrm{CH}$ & MMAS & 0,1327 & 1 & 0,1734 & 6 & 0,2345 & 6 & 0,2956 & 6 & 0,3363 & 6 \\
\hline & \multirow{3}{*}{ USA } & \multirow{3}{*}{$\begin{array}{c}\text { Velayudhan et al. } \\
\text { (2007) }\end{array}$} & $\mathrm{CH}$ & ACO MP & 0,1589 & 7 & 0,1438 & 5 & 0,1211 & 5 & 0,0985 & 5 & 0,0834 & 4 \\
\hline & & & $\mathrm{CH}$ & ACO SLR & 0,1510 & 6 & 0,1350 & 4 & 0,1108 & 4 & 0,0867 & 3 & 0,0706 & 3 \\
\hline & & & $\mathrm{CH}$ & ACO MLR & 0,1467 & 5 & 0,2008 & 7 & 0,2819 & 7 & 0,3631 & 7 & 0,4172 & 7 \\
\hline \multirow{11}{*}{$\begin{array}{l}\text { KroA100; } \\
\text { (1)MC; } \\
\text { (2)NT }\end{array}$} & \multirow{2}{*}{ Colombia } & \multirow{2}{*}{ Pérez (2011a) } & PSis & SCA_2opt_r & 0,08983 & 6 & 0,0722 & 3 & 0,0456 & 2 & 0,0191 & 2 & 0,00146 & 2 \\
\hline & & & PSis & SCA_2opt & 0,09048 & 7 & 0,0726 & 4 & 0,0457 & 3 & 0,0189 & 1 & 0,00100 & 1 \\
\hline & \multirow{2}{*}{$\begin{array}{l}\text { Bélgica y } \\
\text { Suiza }\end{array}$} & \multirow{2}{*}{$\begin{array}{c}\text { Dorigo y } \\
\text { Gambardella } \\
(1997)\end{array}$} & $\mathrm{CH}$ & ACS & 0,08604 & 1 & 0,0757 & 5 & 0,0602 & 8 & 0,0446 & 8 & 0,03428 & 8 \\
\hline & & & $\mathrm{AG}$ & $\mathrm{GA}$ & 0,08798 & 5 & 0,2169 & 11 & 0,4102 & 11 & 0,6036 & 11 & 0,73247 & 11 \\
\hline & \multirow{3}{*}{ USA } & \multirow{3}{*}{$\begin{array}{c}\text { Velayudhan et al. } \\
\text { (2007) }\end{array}$} & $\mathrm{CH}$ & ACO MP & 0,10302 & 11 & 0,083 & 9 & 0,0529 & 7 & 0,0229 & 7 & 0,00284 & 3 \\
\hline & & & $\mathrm{CH}$ & ACO SLR & 0,09796 & 10 & 0,0789 & 8 & 0,0504 & 6 & 0,0219 & 6 & 0,00284 & 3 \\
\hline & & & $\mathrm{CH}$ & ACO MLR & 0,09513 & 9 & 0,0767 & 7 & 0,049 & 5 & 0,0213 & 4 & 0,00284 & 3 \\
\hline & & & $\mathrm{AG}$ & FRAG_GA & 0,08604 & 1 & 0,07 & 2 & 0,0459 & 4 & 0,0218 & 5 & 0,00569 & 7 \\
\hline & Libia & Sallabi y El- & $\mathrm{AG}$ & $\begin{array}{l}\text { SWAP_- } \\
\text { GATSP }\end{array}$ & 0,08694 & 4 & 0,0767 & 6 & 0,0612 & 9 & 0,0458 & 9 & 0,03556 & 9 \\
\hline & & & $\mathrm{AG}$ & OX_SIM & 0,09056 & 8 & 0,108 & 10 & 0,1342 & 10 & 0,1603 & 10 & 0,17778 & 10 \\
\hline & & & $\mathrm{AG}$ & IGA & 0,08604 & 1 & 0,0695 & 1 & 0,0446 & 1 & 0,0198 & 3 & 0,00323 & 6 \\
\hline & Colombia & Pérez $(201$ & PSis & SCA_2opt_r & 0,16763 & 5 & 0,1345 & 1 & 0,0849 & 1 & 0,0353 & 1 & 0,00224 & 2 \\
\hline & Covinista & (2) (2017a) & PSis & SCA_2opt & 0,17292 & 6 & 0,1386 & 2 & 0,087 & 2 & 0,0355 & 2 & 0,00112 & 1 \\
\hline & Ching & Wang y Wang & $\mathrm{CH}$ & MNACO & 0,16485 & 1 & 0,1544 & 3 & 0,1386 & 3 & 0,1229 & 3 & 0,11235 & 3 \\
\hline (1)MC; & Conia & $(20000)$ & $\mathrm{CH}$ & MMAS & 0,16490 & 4 & 0,2138 & 6 & 0,2871 & 6 & 0,3604 & 6 & 0,40930 & 6 \\
\hline (2)TM & China & $\begin{array}{l}\text { Luo, Yang y Li } \\
\text { (2008) }\end{array}$ & Otros & SFLA & 0,16485 & 2 & 0,1675 & 4 & 0,1716 & 4 & 0,1756 & 4 & 0,17825 & 4 \\
\hline & Serbia & $\begin{array}{l}\text { Teodorovic } \\
\text { (2008) }\end{array}$ & Otros & $\mathrm{BCO}$ & 0,16485 & 3 & 0,1912 & 5 & 0,2308 & 5 & 0,2704 & 5 & 0,29674 & 5 \\
\hline & Colombir & Pérez (2011) & PSis & SCA_2opt_r & 0,18893 & 1 & 0,1515 & 1 & 0,0954 & 1 & 0,0393 & 2 & 0,00188 & 2 \\
\hline & & न & PSis & SCA_2opt & 0,19143 & 2 & 0,1532 & 2 & 0,0958 & 2 & 0,0385 & 1 & 0,00023 & 1 \\
\hline (1)MC; & & & ES & CSA & 0,20719 & 4 & 0,2455 & 5 & 0,303 & 5 & 0,3604 & 5 & 0,39876 & 5 \\
\hline (2)NT & $\begin{array}{l}\text { Repúbica de } \\
\text { Korea }\end{array}$ & $\begin{array}{c}\text { Jeong, Kim y Lee } \\
\text { (2009) }\end{array}$ & ES & TFSA & 0,20381 & 3 & 0,2252 & 4 & 0,2573 & 4 & 0,2894 & 4 & 0,31080 & 4 \\
\hline & & & ES & TRFSA & 0,20865 & 5 & 0,2246 & 3 & 0,2485 & 3 & 0,2724 & 3 & 0,28832 & 3 \\
\hline & Colombia & Pérez (2011) & PSis & SCA_2opt_r & 0,19319 & 1 & 0,1547 & 1 & 0,0968 & 1 & 0,039 & 1 & 0,00048 & 2 \\
\hline & - & & PSis & SCA_2opt & 0,19575 & 2 & 0,1566 & 2 & 0,0979 & 2 & 0,0392 & 2 & 0,00012 & 1 \\
\hline (1)CM; & & & $A G$ & AG serial & 0,20403 & 5 & 0,258 & 5 & 0,3391 & 5 & 0,4201 & 5 & 0,47412 & 5 \\
\hline & Brasil & $\begin{array}{l}\text { Dos Santos } \\
\text { (2009) }\end{array}$ & $\mathrm{AG}$ & AG paralelo & 0,20369 & 4 & 0,2168 & 4 & 0,2366 & 4 & 0,2563 & 4 & 0,26946 & 4 \\
\hline & & & $A G$ & AG grupo & 0,20334 & 3 & 0,2138 & 3 & 0,2296 & 3 & 0,2453 & 3 & 0,25582 & 3 \\
\hline
\end{tabular}

MC: Mejor costo; TM; Tiempo medio; NT: Número de tours; CM: Costo medio; Psis: Pensamiento sistémico; CH: Colonia de hormigas, ES: Enfriamiento simulado; AG: Algoritmos genéticos.

Tabla 6. Posiciones clave en la comparación con metaheurísticas.

\begin{tabular}{cccccc}
\hline Métodos & $\begin{array}{c}\text { Grupos } x \\
\text { escenarios }\end{array}$ & 1er puesto & 2do puesto & 3ro puesto & $\begin{array}{c}\text { Último o } \\
\text { penúltimo puesto }\end{array}$ \\
\hline SCA_2opt_r & 25 & 12 & 9 & 2 & 1 \\
SCA_2opt & 25 & 8 & 12 & 1 & 1 \\
\hline
\end{tabular}

Tabla 7. Distribución de las posiciones clave en la comparación con metaheurísticas ante eficacia 100\% y 80\%.

\begin{tabular}{|c|c|c|c|c|c|c|c|}
\hline Escenario & Métodos & Casos & $\begin{array}{c}1 \mathrm{er} \\
\text { puesto }(\%)\end{array}$ & $\begin{array}{c}2 \mathrm{do} \\
\text { puesto }(\%)\end{array}$ & $\begin{array}{c}\text { 3ro } \\
\text { puesto }(\%)\end{array}$ & $\begin{array}{c}\text { Último o } \\
\text { penúltimo } \\
\text { puesto }(\%)\end{array}$ & Otros $(\%)$ \\
\hline \multirow{2}{*}{$\begin{array}{l}\text { Eficacia } 100 \% \text {, } \\
\text { eficiencia } 0 \%\end{array}$} & SCA_2opt_r & 5 & 40 & 0 & 20 & 20 & 20 \\
\hline & SCA_2opt & 5 & 0 & 40 & 0 & 20 & 40 \\
\hline \multirow{2}{*}{$\begin{array}{l}\text { Eficacia 80\%, } \\
\text { eficiencia 20\% }\end{array}$} & SCA_2opt_r & 5 & 80 & 0 & 20 & 0 & 0 \\
\hline & SCA_2opt & 5 & 0 & 80 & 0 & 0 & 20 \\
\hline
\end{tabular}


Siendo $\mathrm{D}_{\mathrm{ijk}}$ el desempeño de la alternativa i en el factor $\mathrm{j}$ cuando fue probada en la instancia $\mathrm{k}$. $\mathrm{DN}_{\mathrm{ijk}}$ es este mismo valor pero normalizado entre 0 y 1.

En la Tabla 4, se detallan los grupos y sus características; para cada grupo, se muestra la matriz de pagos, tanto con los valores de eficacia y de eficiencia originales, como normalizados.

Considerando $\mathrm{P}_{\mathrm{j}}$ como el peso del factor $\mathrm{j}$ (eficacia, eficiencia) y $\mathrm{DN}_{\mathrm{ijk}}$ el desempeño, normalizado, de la alternativa $i$ en el factor $j$ cuando es probada en una instancia k determinada, es posible estimar el desempeño global, normalizado, de la i-ésima altenativa de decisión $\left(\mathrm{DN}_{\mathrm{i} k}\right.$ ) mediante la Ecuación 2.

$$
D N_{i k}=\sum_{j=1}^{2} D N_{i j k} \times P_{j} \quad \forall_{i, k}
$$

En la Tabla 5 se muestran los resultados finales para cada grupo de $\mathrm{k}=5$ instancias. Allí se presenta tanto el $\mathrm{DN}_{\mathrm{ik}}$, como el puesto ocupado por los diferentes métodos.

A partir de la Tabla 5, se calculó el número de casos en que las dos heurísticas propuestas ocuparon diversos puestos, delimitados a las siguientes categorías de interés: $1^{\circ}, 2^{\circ}, 3^{\circ}$, el último o penúltimo, y otros (ver Tabla 6).

Con base en esta información, puede verse que el SCA_2opt_r, considerando los 25 casos comparativos (cinco escenarios por cinco grupos de instancias), en la mayoría de ellos ocupó el primero o el segundo puesto (84\%). Similar ocurrió para el SCA_2opt, con el $80 \%$ de los casos. Esto refleja un desempeño prometedor de los métodos propuestos. Haciendo énfasis en el escenario extremista de eficacia 100\% y en el de un decisor que ve este factor como vital $(80 \%)$ sin llegar al extremo de nula la eficiencia, en la Tabla 7 se observa la distribución de los puestos ocupados por los dos métodos propuestos.

En términos globales, consolidando los resultados para los escenarios: eficacia extremista (100\%) y vital (80\%), el SCA_opt_r, de los 10 casos comparativos, en seis de ellos ocupó el primer lugar. El SCA_2opt tuvo este mismo resultado, pero ocupando el segundo lugar. Esto refleja lo promisorios que pueden ser los métodos propuestos considerando diversos tipos de decisores que se tienen en la realidad.

\section{Conclusiones}

El estudio exploró la posible viabilidad de que el pensamiento sistémico (PS) sea tomado como un nuevo campo de inspiración para el desarrollo de noveles métodos de optimización combinatoria, probándolo en el problema benchmark: el TSP. Esta iniciativa partió del análisis sistémico de la clásica regla de decisión VMC, para la cual se encontró que sus comportamientos son viables de comprender desde las leyes de la quinta disciplina, entre ellas: causa y efecto están distantes en tiempo y en espacio, las soluciones fáciles llevan al mismo lugar, y los problemas de hoy derivan de las soluciones del ayer. La observación de estas leyes del PS llevó a identificar que en la práctica del VMC, ilustrada en el caso de don Pablo, subyace el arquetipo soluciones rápidas que fallan. Para abordar este patrón, el estudio se apoyó en otra de las leyes del PS, consistente en la búsqueda de un punto de apalancamiento que permita mejorar globalmente el sistema. Fruto de esta búsqueda, surgió la estrategia Sacrificio cortoplacista adaptativo (SCA), la cual se basa en que a veces es necesario hacer sacrificios en el corto plazo, con el ánimo de tener un futuro mejor. Este heurismo se materializó, desde el punto de vista algorítmico, en que en algún momento de la práctica del VMC, el siguiente desplazamiento no se realice a la ciudad inmediatamente cercana disponible, sino que se renuncie a ella para trasladarse hacia la segunda más cercana disponible $\mathrm{y}$, a partir de este cambio, se continúe el VMC. Esta estrategia, atendiendo a una de las tendencias arrojadas por la revisión de literatura, llevó a complementarlo con la búsqueda local 2opt, dando lugar al SCA_2opt.

El SCA_2opt condujo, a través de su estudio, a una nueva versión del mismo, el SCA_2opt_r. Este último contiene la alteración de uno de los parámetros de la búsqueda local, con posibilidad de variación en un rango de 0 a 1 . Para estos dos métodos, se corroboró que el favorable desempeño no se originó en la búsqueda local, ni en el azar, sino en el SCA. Los dos métodos propuestos fueron comparados con 19 metaheurísticas en las categorías: colonia de hormigas, algoritmos genéticos, enfriamiento simulado y otros, obteniendo resultados promisorios en el binomio eficacia y eficiencia. Cabe destacar que ninguna de las heurísticas propuestas: SCA_2opt y SCA_2opt_r incorpora fenómenos aleatorios.

Este trabajo muestra, además, un procedimiento para comparar métodos heurísticos, vinculando análisis multicriterio. Esto debido a que dos de las propiedades deseables en un método de este tipo son eficacia y eficiencia, los cuales, en casos, suelen verse contradictorios: cuando se obtiene eficacia destacada, generalmente es a costa de pérdida de eficiencia. El análisis multicriterio, además, posibilita vincular los intereses del decisor. Dicho análisis se realizó a la luz de cinco escenarios: dos extremistas, en los cuales el decisor deposita todo su interés (100\%) en uno de los factores, dejando el otro nulo; otros dos escenarios consideraron el principio de Pareto (80\%-20\% y viceversa), y el otro representó aquel 
decisor que mantiene un equilibrio 50\%-50\%. El procedimiento propuesto destaca las posiciones que ocupan los algoritmos a la luz de los cinco escenarios y posibilita analizar la sensibilidad de los mismos ante cambios en el peso de los factores, brindando características de robustez a los métodos comparados. Este procedimiento puede constituirse en una alternativa para la comunidad académica, en el sentido de vincular otros aspectos a la hora de juzgar sobre el desempeño de los nuevos métodos heurísticos a proponer.

En la comparación de los dos métodos propuestos contra las 19 metaheurísticas, las fuentes bibliográficas desde las que se extrajo la información referente, provienen de trabajos realizados por autores afiliados a instituciones de los ámbitos: USA, Bélgica, Suiza, Brasil, China, Serbia y República de Korea. Esto brinda una idea del enfoque asumido para comparar el desempeño de las dos heurísticas propuestas, abordando referentes internacionales.

En términos generales, el estudio se constituye en un primer aporte que refleja la viabilidad de emplear el pensamiento sistémico como un pertinente campo de inspiración para el desarrollo de nuevos métodos de optimización heurística, dado que ofrece evidencia objetiva sobre el diseño y desarrollo de un algoritmo heurístico, basado en el análisis sistémico del "Vecino más cercano", capaz de arrojar soluciones efectivas para instancias simétricas TSP.

\section{Trabajo futuro}

Se espera que este artículo logre motivar e inspirar a otros investigadores y estudiantes para dar respuesta, como mínimo, a las siguientes preguntas: ¿qué valor del parámetro de la búsqueda local 2_opt, empleado en este estudio, optimiza el desempeño de la heurística SCA_2opt_r?,¿qué efecto tiene en el desempeño de los actuales SCA_2opt y SCA_2opt_r, el emplear buscadores locales k_opt, superiores a $k=2$ ?, ¿en el SCA, el hecho de trasladar al viajero hacia la $\mathrm{k}$ ciudad inmediatamente más cercana, con $\mathrm{k}$ superiores a 2, tiene un desempeño diferente a realizarlo de acuerdo a la política actual (segunda ciudad disponible, más cercana)?, de ser así, ¿cuál k conlleva al mejor desempeño de la heurística?; ¿existen otras heurísticas cuyo comportamiento sea viable analizar desde el pensamiento sistémico?, de encontrarse, ¿qué arquetipos sistémicos subyacen en ellas?

\section{Referencias}

BAHILL, T.; GISSING, B. Re-evaluating systems engineering concepts using systems thinking. IEEE Transactions on
Systems, Man and Cybernetics Part C: Applications and Reviews, v. 28, n. 4, p. 516-527, 1998. http://dx.doi. org/10.1109/5326.725338

BERBIELA, J. La inteligencia artificial aplicada a la gestión de la tesorería. Revista Real Academia de Ciencias Exactas, Físicas y Naturales, v. 92, n. 4, p. 435-439, 1998. Monográfico: Problemas complejos de decisión.

BENTLEY, J. Fast algorithms for geometric traveling salesman problems. ORSA Journal on Computing, v. 4, n. 4, p. 387-411, 1992. http://dx.doi.org/10.1287/ijoc.4.4.387

CARTER, A.; RAGSDALE, C. A new approach to solving the multiple traveling salesperson problem using genetic algorithms. European Journal of Operational Research, v. 175, n. 1, p. 246-257, 2006. http://dx.doi. org/10.1016/j.ejor.2005.04.027

DANTZIG, G.; FULKERSON, R.; JOHNSON, S. Solution of a large-scale traveling-salesman problem. Journal of the operations research society of America, v. 2, n. 4, p. 393410, 1954.

DISTEFANO, M.; HAARTH, R.; IRIARTE, E. Modelación de sistemas tecnológicos en la formación básica de los ingenieros. In: CONGRESO TECNOLOGÍAS APLICADAS A LA ENSEÑANZA DE LA. ELECTRÓNICA - TAEE, 2006, Madrid. Actas... Universidad Nacional de Cuyo, 2006.

DORIGO, M.; GAMBARDELLA, M. Ant-Q: A reinforcement learning approach to the traveling salesman problem. In: INTERNATIONAL CONFERENCE ON MACHINE LEARNING, 1995, Tahoe City. Proceedings... Tahoe City, 1995. p. 252-260.

DORIGO, M.; GAMBARDELLA, M. Ant colony system: A cooperative learning approach to the traveling salesman problem. IEEE Transactions on Evolutionary Computation, v. 1, n. 1, p. 53-66, 1997. http://dx.doi. org/10.1109/4235.585892

DOS SANTOS, J. P. Q. Uma implementação paralela híbrida para o problema do caixeiro viajante usando algoritmos genéticos, GRASP e aprendizagem por reforço. 73 f. Dissertação (Mestrado em Engenharia Elétrica)-Universidade Federal do Rio Grande do Norte, Natal, 2009.

FLO0D, M. The Traveling Salesman Problem. Operations Research, v. 4, p. 61-75, 1956. http://dx.doi.org/10.1287/ opre.4.1.61

GOLDEN, B. et al. Approximate traveling salesman algorithms. Operations research, v. 28, p. 694-711, 1980. http:// dx.doi.org/10.1287/opre.28.3.694

GÓMEZ, M.; LEÓN, F. Planeación de Rutas de Distribución utilizando el Algoritmo Heurístico 2-Optimal: Implementación Computacional. INVURNUS, v. 6, n. 1, p. 15-20, 2011.

HANSEN, P.; MLADENOVIC, N. Variable neighbourhood search. In: GLOVER, F.; KOCHENBERGER, G. A. (Eds.). Handbook of Metaheuristics. Kluwer, 2003.

JEONG, S.; KIM, K.; LEE, Y. The efficient search method of simulated annealing using fuzzy logic controller. Expert Systems with Applications, v. 36, p. 7099-7103, 2009. http://dx.doi.org/10.1016/j.eswa.2008.08.020

JÜNGER, M.; REINELT, G.; RINALDI, G. The Traveling Salesman Problem. In: BALL, M. O. et al. (Eds.). Handbook in Operations Research and Management Science. NorthHolland, 1995. v. 7, p. 225-330.

KARABOGA, D.; GORKEMLl, B. A combinatorial Artificial Bee Colony algorithm for traveling salesman 
problem. In: INTERNATIONAL SYMPOSIUM ON INNOVATIONS IN INTELLIGENT SYSTEMS AND APPLICATIONS - INISTA, 2011, Istanbul. Proceedings... Istanbul, 2011. p. 50-53.

LETELIER, M. et al. Competencias sustentables para el desempeño profesional en ingeniería. Revista Facultad de Ingeniería - Universidad de Tarapacá, v. 13, n. 2, p. 9196, 2005.

LIU, Y. A hybrid scatter search for the probabilistic traveling salesman problem. Computers and Operations Research, v. 34, n. 10, p. 2949-2963, 2007. http://dx.doi. org/10.1016/j.cor.2005.11.008

LUO, X.; YANG, Y.; Ll, X. Solving TSP with Shuffled Frog-Leaping Algorithm. In: INTERNATIONAL CONFERENCE ON INTELLIGENT SYSTEMS DESIGN AND APPLICATIONS, 2008, Kaohsiung City. Proceedings... IEEE, 2008. p. 228-232.

MARTI, R. Procedimientos metaheurísticos en optimización combinatoria. Matemátiques. v. 1, n. 1, p. 1-60, 2003.

MORALES, R. et al. Paralelización de un Algoritmo de Búsqueda Local Iterada para el Problema del Agente Viajero. In: CONGRESO INTERNACIONAL DE CÓMPUTO EN OPTIMIZACIÓN Y SOFTWARE - CICOS, 2011, Cuernavaca. Memorias... UAEM México, 2011.

NGUYEN, $H$. et al. Implementation of an effective hybrid GA for large-scale traveling salesman problems. IEEE Transactions on Systems, Man, and Cybernetics, Part B: Cybernetics, v. 37, n. 1, p. 92-99, 2007. http://dx.doi. org/10.1109/TSMCB.2006.880136

PÉREZ, J. Heurística inspirada en el análisis sistémico del "Vecino más cercano", para solucionar instancias simétricas TSP, empleando una base comparativa multicriterio. 2011. 130 f. Tesis (Magíster en ingeniería de sistemas)-Facultad de Minas, Universidad Nacional de Colombia, Medellín, 2011a.
PÉREZ, J. Modelación lineal en ingeniería industrial: Una mirada sistémica. Medellín: Editorial Universidad de Antioquia, 2011b. (Colección Ciencia y Tecnología).

REINELT, G. TSPLIB. A traveling salesman problem library. ORSA Journal on Computing, v. 3, n. 4, p. 376384, 1991. http://dx.doi.org/10.1287/ijoc.3.4.376

SALLABI, O.; EL-HADDAD, Y. An Improved Genetic Algorithm to Solve the Traveling Salesman Problem. World Academy of Science, Engineering and Technology, n. 52, p. 471474, 2009.

SENGE, P. La Quinta Disciplina: El arte y la práctica de la organización abierta al aprendizaje. Buenos Aires: Ediciones Granica S.A., 1998. 490 p.

TEODOROVIC, D. Swarm intelligence systems for transportation engineering: Principles and applications. Transportation Research Part C, n. 16, p. 651-667, 2008. http://dx.doi.org/10.1016/j.trc.2008.03.002

VELAYUDHAN, P. et al. Empirical Analysis of Randomness in Ant Colony Optimization Algorithms Applied to the Traveling Salesman Problem. International Journal of Information Systems for Logistics and Management, v. 2, n. 2, p. 69-76, 2007.

WANG, J.; WANG, W. Research on ACO with Multiple Nests' Cooperation for Narrow TSP. In: INTERNATIONAL CONFERENCE ON BIO-INSPIRED COMPUTING: THEORIES AND APPLICATIONS, 3., 2008, Adelaide. Proceedings... 2008. IEEE, 2008. p. 143-147.

WHITLEY, D.; HAINS, D.; HOWE, A. A Hybrid Genetic Algorithm for the Traveling Salesman Problem Using Generalized Partition Crossover. In: PARALLEL PROBLEM SOLVING FROM NATURE - PPSN, 11., 2010, Poland. Lecture Notes in Computer Science, v. 6238, p. 566575, 2010.

\title{
Sacrifice short-term adaptive 2-opt (SCA_2opt): A heuristic inspired by systems thinking
}

\begin{abstract}
Outlining two novice heuristics for the symmetric TSP, inspired systemic thinking: Sacrifice short-term adaptive 2-opt (SCA2opt) and SCA_2opt_r. These arise from the systemic analysis of the rule of decision, nearest neighbour, identifying the archetype "Counterproductive solutions". SCA relies on that agent traveler, renounces at any given time immediately to a nearby city, and moves towards the second nearest available city. From there, it continues with the nearest neighbour rule. Each is the SCA (global search) operates a local search 2opt. Where as the binomial effectiveness and efficiency, the two new heuristics are shown promising in comparison multicriteria against 19 metaheuristics. It is evident that systemic thinking is a field of inspiration viable for the development of combinatorial optimization methods; emerging questions for future developments are expressed, enabling to continue to integrate elements of the classical optimization with the systemic thought; areas traditionally seen as antagonistic, but whose dialogue is favourable in this article.
\end{abstract}

Keywords

Sacrifice short-term adaptive. TSP. Metaheuristics. Multicriteria analysis. Systems thinking. 